\title{
Persönliche Lernumgebungen: Grundlagen, Möglichkeiten und Herausforderungen eines neuen Konzepts
}

von Sandra Schaffert (Salzburg Research) und

Marco Kalz (Centre for Learning Sciences and Technologies, Open University of the Netherlands)

Selbstorganisiertes Lernen · Personal Learning Environment · Persönliche Lernumgebung - Social Software - Bildungstechnologie · LernManagement-System

Wie gelernt und gelehrt wird, ist nicht allein eine Frage der verwendeten Technologie, wird jedoch von ihr beeinflusst. Die Entwicklung und der wachsende Erfolg von partizipativen Anwendungen im Internet (englisch: "Social Software«) wie Wikis und Weblogs führten zur Innovation technologiegestützten Lernens: Unter dem Begriff der »Persönlichen Lernumgebung « (englisch: »Personal Learning Environment«, abgekürzt PLE) setzt sich ein neues Konzept deutlich von den traditionellen Realisierungen von E-Learning, wie z. B. den Lern-Management-Systemen, ab. Im Fokus der »Persönlichen Lernumgebung « steht der Lernende, der sich selbst Webinhalte, Lernressourcen und Lernwerkzeuge so arrangiert und sie so nutzt, dass sie sein persönliches Wissensmanagement und Lernen unterstützen. Der Beitrag beschreibt die Genese und Besonderheiten des Konzepts und bisherige Realisierungen. Dabei werden auch Abgrenzungen zu anderen, traditionellen technologischen Konzepten diskutiert sowie verwandte (didaktische) Methoden beschrieben.

Schlagworte

Überblick

\section{Einleitung?}

Social Software als technologische Grundlage für PLE

Das Konzept PLE

Realisierungen von PLE

Service-Integration in vorhandene Lernumgebungen

Integrierte Oberflächen

PLE als Framework

Das Konzept der PLE im Vergleich mit traditionellen

technologischen Lehr- und Lernkonzepten

5.1 Vergleich mit vorhandenen technologischen Konzepten

5.2 Vergleich mit Lern-Management-Systemen

6 Verwandte (didaktische) Methoden und Ansätze

6.1 PLE und persönliches Wissensmanagement

6.2 PLE und E-Portfolio

6.3 PLE und »Communities of Practice«

7 Ausblick

Literaturhinweise 


\section{$1 \quad$ Einleitung}

Der technologische Fortschritt beeinflusst die Möglichkeiten und Methoden von Lernen und Lehre erheblich. Technologiegestütztes Lernen mit dem Internet findet nicht nur in Form von virtuellen Seminaren oder in Form anderer formaler Lernarrangements statt: Viele nutzen Computer und Internet auch beiläufig oder informell zum Lernen bzw. zur Kompetenzentwicklung. An vielen Bildungseinrichtungen und Arbeitsplätzen sind der Computer und das Internet die zentralen Medien für das Lernen.

Dabei lässt sich beobachten, dass trotz aller Euphorie über didaktische Innovationen bei der Einführung neuer Medientechnologie die Neigung besteht, vorhandene pädagogische Paradigmen und Praktiken zu imitieren bzw. mit den neuen Technologien neu zu implementieren, anstatt diese zum Anlass für neue Lern- und Lehrszenarien zu nehmen. Traditionelle didaktische Modelle, in denen z. B. die Lehrperson als Wissensträger ihr Wissen auf die Lernenden einfach übertragen muss, sind in Form von Computer Based Trainings (WBT) oder Web Based Trainings (WBT) in großem Umfang realisiert worden.

Neue Rolle Das Konzept der »Persönlichen Lernumgebung « rollt die Frage nach dem des Lernenden Lernenden jedoch neu auf. Im Unterschied zu den oben genannten Konzepten rückt es radikal den selbst gesteuerten und aktiven Lerner in den Fokus. Das Konzept der Persönlichen Lernumgebung heißt im Englischen »Personal Learning Environments« und wird im Folgenden mit »PLE«abgekürzt.

Auf den ersten Blick wird man mit dem Begriff »Persönliche Lernumgebung « nicht zwangsläufig eine neue Variante des internetgestützten Lernens assoziieren: Der Begriff zielt zunächst einmal darauf ab, dass es sich hier um die individuelle, nach persönlichen Interessen und Bedürfnissen ausgerichtete, also personalisierte Umgebung handelt, in der Lernende ihr persönliches Wissensmanagement und ihre eigene Weiterbildung organisieren.

Tatsächlich verbirgt sich hinter PLE jedoch ein neues technologisches Konzept für die Unterstützung von Lernenden, dessen Grundlage die neuen Social-Software-Anwendungen sind. Nach einer kurzen Einführung in diese beschreibt dieser Beitrag das Konzept PLE und seine Genese sowie erste Realisierungen von PLE. Um die Besonderheiten des PLE-Konzepts zu veranschaulichen, wird es im Anschluss mit vorhandenen technologischen Konzepten, insbesondere mit Lern-Management-Systemen verglichen. Zudem werden verwandte (didaktische) Methoden und Ansätze wie die E-Portfolio-Methode beschrieben.

\section{Social Software als technologische Grundlage für PLE}

Das Konzept der PLE steht im engen Zusammenhang mit der Entwicklung und Existenz unterschiedlicher Applikationen, die man unter der Kategorie »Social Software« zusammenfasst. Darunter werden Software-Anwendungen verstanden, die Menschen verbinden und Kommunikation und Zusammenarbeit ermöglichen (SCHAFFERT 2007). In den letzten Jahren hat sich hierbei vieles getan, die Web-basierten Tools machen die Zusammenarbeit und Kommunikation immer leichter. Dabei kann man die folgenden Anwendungsbereiche von Social Software unterscheiden: 
Kommunikation (Diskussions- und Kommunikationsanwendungen wie Weblogs, Microblogging-Applikationen),

kollaborative Entwicklung (gemeinsame Erstellung von Texten und anderen Produkten z. B. in Wiki-Systemen),

kollaborative Anreicherung von Inhalten (z. B. in Form von Social Bookmarking oder Social Tagging),

Vernetzung (Vernetzung von Geschäftskontakten und Bekanntschaften, wie es LinkedIn, Xing, studiVZ ermöglichen, aber auch Anwendungen, die es ermöglichen, Personen mit ähnlichen Interessen zu finden).

Durch die Verbreitung von neuen benutzerfreundlichen Applikationen hat sich die Partizipation und Mitgestaltung im Internet erheblich erleichtert. Neue Werkzeuge, Anwendungen und sogenannte Web-Services machen es immer leichter, Inhalte und Webseiten zu organisieren. In den folgenden Abschnitten geben wir einen kurzen Einblick in einige dieser Social-SoftwareTechnologien und Anwendungen und zeigen, in welcher Weise diese auch im Bildungsbereich eingesetzt werden.

So ermöglicht das Wiki-System, auf dem u. a. die Online-Enzyklopädie Wikipedia beruht, eine vergleichsweise einfache kooperative Erstellung von Webseiten. Wiki-Systeme wurden daher schnell im Bildungswesen eingesetzt, weil sie für das kollaborative Erstellen von Beiträgen, beispielsweise im Rahmen von Projektarbeiten, Seminararbeiten oder Glossaren, derzeit die beste Lösung darstellen. Auch viele wichtige Projekte zum gemeinsamen Erstellen von Unterrichtsmaterialien nutzen die Wiki-Technologie, beispielsweise das deutschsprachige ZUM-Wiki oder die Plattform Wikieducator.org.

Auch Weblogs, in denen Internetnutzer regelmäßig ihre persönliche Sicht auf die Welt veröffentlichen, werden als Social Software verstanden: Durch ihre Kommentarfunktion und Verweise auf Einträge anderer Weblog-Autoren unterstützen sie den sozialen Austausch und spannen dadurch oft weltweite Diskursnetzwerke auf. Während Weblogs zu Beginn vonseiten der Wirtschaft oft belächelt wurden, werden sie heute ernst genommen: Beispielsweise wurde erkannt, dass Weblogs für Viral-Marketing nutzbar sind und dass sie mit negativen Kundenberichten dem Ruf eines Unternehmens erheblich schaden können. Darüber hinaus sind sie auch Kernelement vieler neuer Varianten interner Wissensmanagement-Lösungen von Unternehmen, wie beispielsweise für SUN Microsystems (HILzENSAUER/SCHAFFER 2008). Auch zu Einsatzmöglichkeiten für Lehr- und Lernzwecke liegen etliche Praxisberichte vor.

Neben diesen beiden Applikationen, die vor allem gute Beispiele für die neuen Möglichkeiten des (kollaborativen) Erstellens von Inhalten sind, gibt es weitere Realisierungen von Social Software, die den Austausch und das gemeinsame Arbeiten ggf. indirekt beeinflussen. Dazu zählt beispielsweise das Social Tagging, bei dem Nutzer eines Services einzelne Webseiten, Fotos oder andere Inhalte »taggen «, also mit Schlagwörtern versehen, und diese wiederum von allen Nutzern einsehbar sind und für eigene Recherchen nutzbar werden. Social Tagging wird insbesondere von Video- und Fotodatenbanken angeboten, weil gerade diese Daten über normale Suchmöglichkeiten (Volltextsuche, Kategorien) kaum ausreichend erschlossen oder recherchierbar sind. Delicious.com ist einer der bekanntesten Services im Bereich des Social Bookmarking. Hiermit können beliebige URLs gemerkt und verschlagwortet werden, was wiederum anderen Nutzern zur Verfügung gestellt werden kann. Auch im Bildungsbereich, insbesondere bei Web-Datenbanken
Social-SoftwareAnwendungen

Wikis

Weblogs

Social Tagging 
und Verzeichnissen von Bildungsressourcen, wird Tagging immer häufiger eingesetzt (siehe z. B. LeMill.net).

RSS-Format Eine wichtige übergreifende technologische Entwicklung, die allen SocialSoftware-Applikationen gemeinsam ist, ist die Portierbarkeit von Daten durch das RSS-Format (RSS ist die Abkürzung für »Really Simple Syndication «). Mit Hilfe des RSS-Formates lassen sich Daten von einem Inhalteanbieter zu einer anderen Website übertragen (dies nennt man Syndikation von Inhalten), bzw. lassen sich so Inhalte aus unterschiedlichen Quellen einfach sammeln (dies nennt man Aggregation). Es lassen sich beispielsweise bei Weblogs (in aller Regel) sogenannte »RSS-Feeds « abonnieren. So kann man neue Informationen der Weblog-Favoriten in eigene, personalisierte Webapplikationen einbinden. »I-Google « oder »Netvibes « sind Beispiele für solche personalisierten Startseiten, bei denen man sich individuelle Feeds zusammenstellen kann. Je nach Weblogs, die man abonniert, kann man sich so über die neuesten Nachrichten oder neue Lerneinheiten z. B. im Business-EnglishWeblog informieren, ohne die Ursprungsquellen selbst einzeln besuchen zu müssen. Auch viele Interessens- und Fachportale binden auf diese Weise externe Quellen in ihr Angebot ein.

Netzwerke und Empfehlungssysteme

Weitere Varianten und neue Entwicklungen von Social Software beschäftigen sich mit den Möglichkeiten, soziale Netzwerke zu knüpfen und zu pflegen (z. B. Angebote wie von Xing.com, studiVZ.de oder Facebook.com). Auch wird daran gearbeitet, die vorhandenen Daten für Empfehlungen für neue passende oder interessante Meldungen oder auch Personen auszuwerten und dies in Empfehlungssystemen zu nutzen. Ein bekanntes Beispiel hierfür sind die Empfehlungen des Online-Buchhändlers Amazon.com: »Personen, die dieses Buch gekauft haben, kauften auch ...«. Ein weiterer Bereich, in dem in letzter Zeit viele Neuerungen vorzufinden sind, ist die Entwicklung von Reputationssystemen: Zum einen deshalb, weil aktive Teilnehmer im Web auch das Interesse haben, dass ihre Aktivität und Kompetenzen in entsprechender Reputation, beispielsweise in Webportalen, sichtbar und nachweisbar wird. Zum anderen sind solche Modelle und das darauf bauende Vertrauen in potenzielle Geschäftspartner, beispielsweise bei Ebay.com, die Grundlage für viele Geschäfte, die unter ansonsten »Unbekannten«stattfinden und über die man nicht, wie früher, anderweitig Erkundigungen einholen konnte.

Diese neuen Möglichkeiten, die mit dem Schlagwort »Social Software« bezeichnet werden, bilden die wesentliche Grundlage für Persönliche Lernumgebungen (PLE) als ein neues Konzept der Organisation und Durchführung des technologiegestützten Lernens mit dem Internet.

\section{Das Konzept PLE}

PLE ist noch jung

Zwar wird das Konzept der Persönlichen Lernumgebungen (PLE) erst seit kurzer Zeit auf einer breiteren Basis diskutiert, jedoch gab es schon vor einigen Jahren erste Ansätze in diese Richtung. Obwohl einige Applikationen bereits früher ähnliche Funktionalitäten wie PLE hatten, waren OLIVIER und LIBER (2001) die Ersten, die diese Idee in einem (überraschenderweise) nicht publizierten Beitrag thematisiert haben. Einige Jahre später waren es die Entwickler der Blogging- und Social-Networking-Plattform »Elgg «, die mit den »Personal Learning Landscapes« ein integriertes Konzept vorgestellt haben, aus 
dem sich dann später das Konzept der »Personal Learning Environments« entwickelt hat.

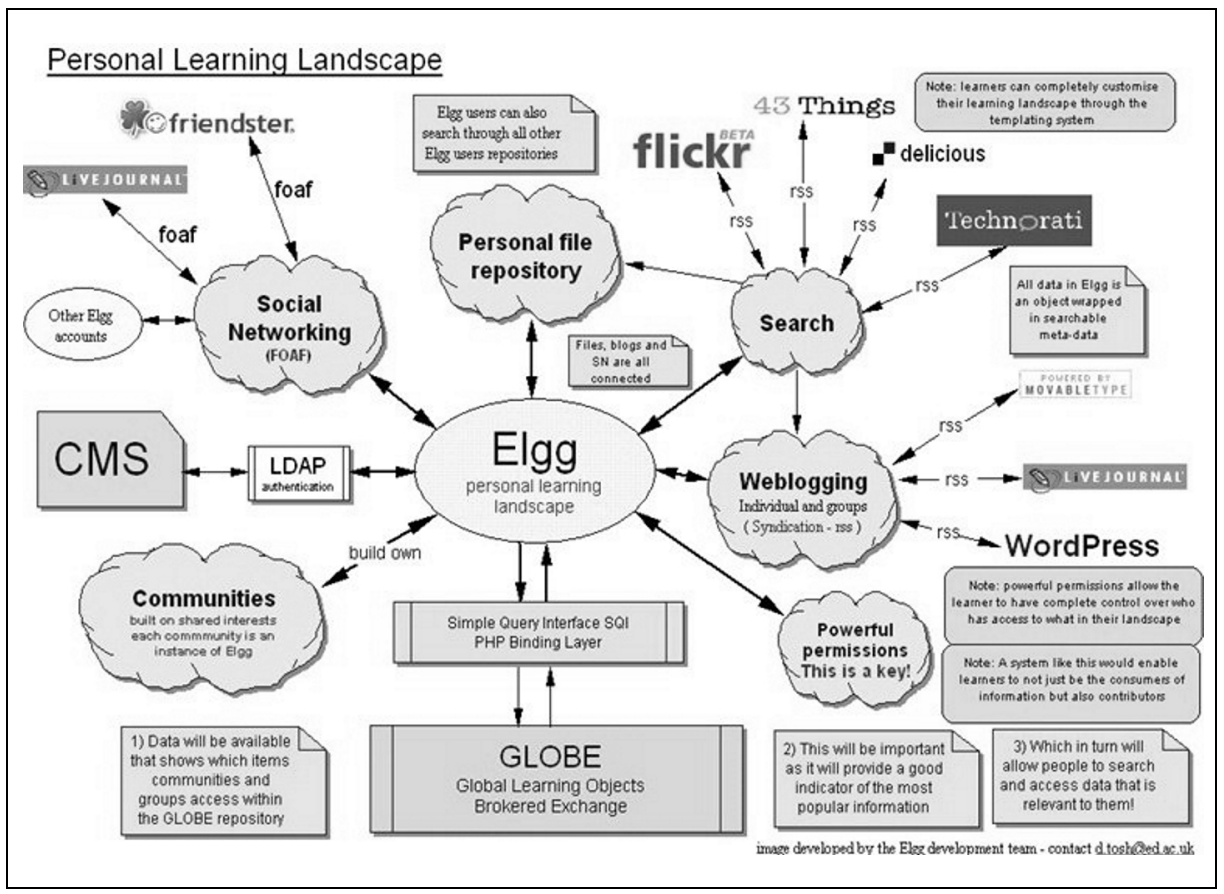

Abb. 1: »Personal Learning Landscape« von Tosh und Werdmuller (2004)

Das PLE-Konzept ist also eine relativ junge Neuentwicklung bzw. -konzeption, die aber in jüngster Zeit intensiv in der E-Learning-Community diskutiert wird. Dies sieht man u. a. daran, dass der Begriff bis zum Jahr 2006 kaum in einer der größten Suchmaschinen gesucht wurde, wie Abbildung 2 zeigt.

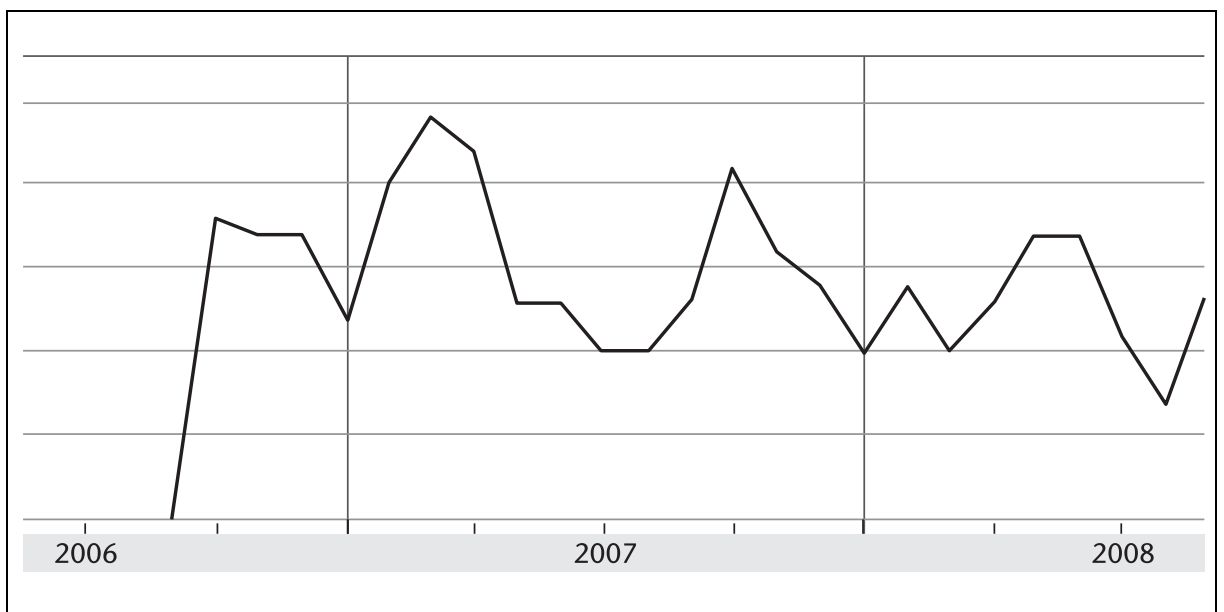

Abb. 2: Häufigkeit der Suche nach »Personal Learning Environment « bei Google (weltweit, seit 2004) nach Google Insights, Stand: 25. 10. 2008

Ein weiterer wichtiger Schritt im Rahmen der Genese des PLE-Konzepts ist die Generalisierung des Konzeptes der »Personal Learning Landscapes« hin zu einer Erweiterung des aktuellen Modells virtueller Lernumgebungen. WiLsoN sieht 2004 in der Zukunft der virtuellen Lernumgebung im Kern die Öffnung hin zur Integration von externen Diensten und Applikationen. Dabei soll 
diese Umgebung Inhalte aus unterschiedlichen externen Quellen importieren können und gleichzeitig auch eigene Produkte publizieren und exportierbar machen.

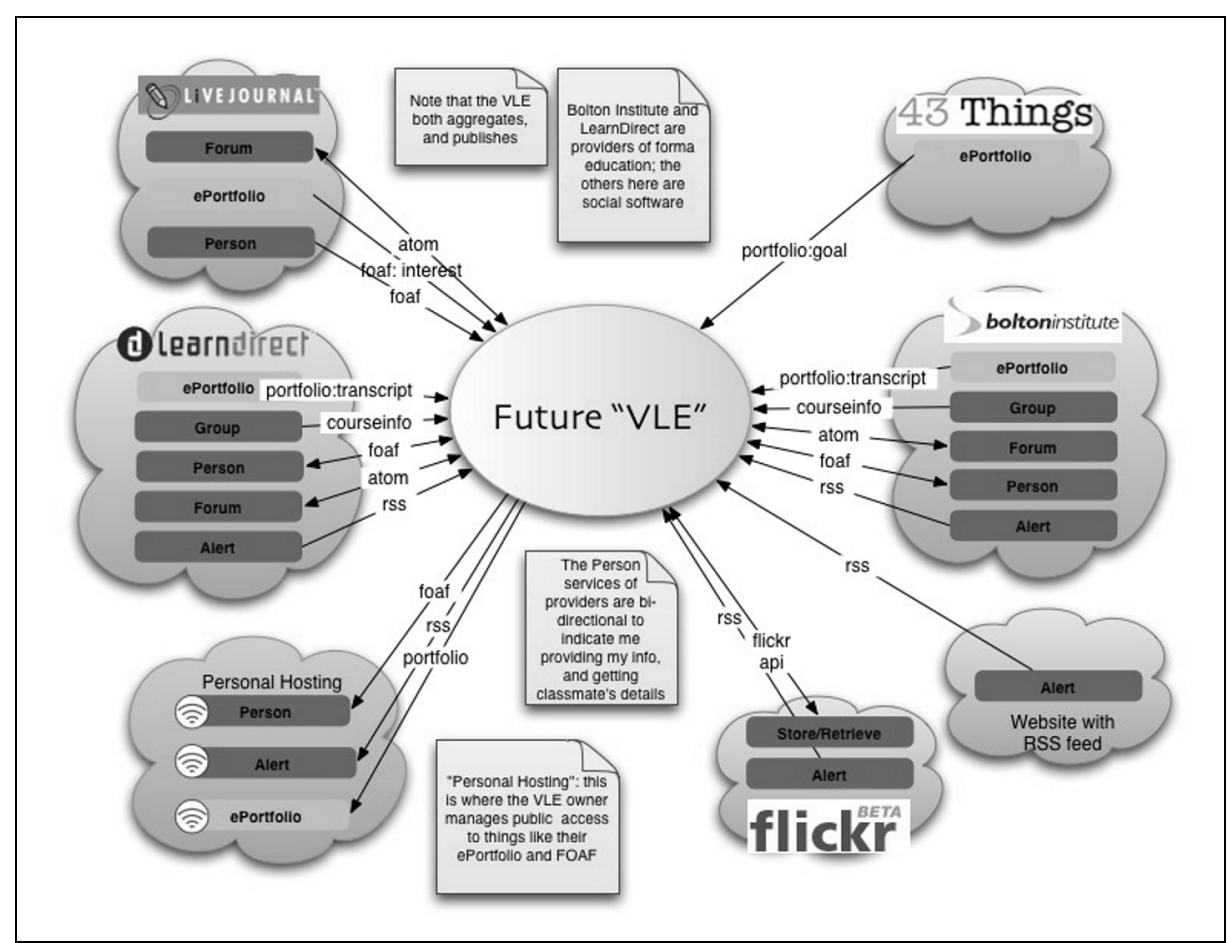

Lernerzentrierter Ansatz

Definition

Technologisches Konzept
Abb. 3: Abbildung einer virtuellen Lernumgebung im Weblog von Scott WiLsoN, die als erste Skizze einer PLE gilt.

Auch wenn es im Detail unterschiedliche Auslegungen des PLE-Konzepts gibt, haben alle Ansätze einen Aspekt gemeinsam: PLE ist ein vorrangig technologisches Konzept, das jedoch wichtige didaktische als auch organisatorische Konsequenzen haben kann. Im Gegensatz zu traditionellen multifunktionalen virtuellen Lernumgebungen, die verschiedene Aspekte in das System integrieren (Studierendenverwaltung, Kommunikations- und Kollaborationswerkzeuge etc.), stellt das PLE-Konzept den Lernenden und seine Aktivitäten und Bedürfnisse in den Mittelpunkt und holt die Werkzeuge und Informationen in das aktuelle favorisierte PLE des Lernenden. Technologisch wird dies u. a. durch sogenannte »serviceorientierte Architekturen (SOA) realisiert sowie durch sogenannte »Web Services«. Diesem Ansatz folgend möchten wir hier unsere Definition von Persönlichen Lernumgebungen formulieren:

Persönliche Lernumgebungen, kurz PLE, sind Lernanwendungen, bei denen Lerner verteilte Online-Informationen,-Ressourcen oder-Kontakte einerseits selbst in ihre PLE integrieren können und andererseits auch ihre im Rahmen der PLE vollzogenen Aktivitäten und deren Produkte in anderen Online-Umgebungen auf der Basis von Standards zur Verfügung stellen können.

PLE ist also weniger ein neues didaktisches Modell oder eine konkrete Methode, die das Lehren oder das Lernen unterstützen kann, als ein technologisches Konzept: Es beschreibt Funktionalitäten, die ein entsprechendes System haben sollte, wenn es als »persönliche Lernumgebung « eingesetzt werden soll. Um eine Lernanwendung zu sein, muss eine PLE die Wissens- und Kompetenzerweiterung von Individuen aktiv unterstützen. Betrachtet man 
die vorzufindenden Verständnisse von PLE, hat der Begriff »persönlich « unterschiedliche Facetten: »Persönlich« betrifft (a) die individuelle Gestaltung der Inhalte und des Layouts, (b) die Unzugänglichkeit für andere (eine PLE sollte privat sein), (c) das Eigentum (eine PLE sollte dem Lernenden gehören) und (d) den Schutz der persönlichen Daten.

Im Gegensatz zu anderen Ansätzen zur Personalisierung von Lernumgebungen, z. B. aus der adaptiven Hypermedia-Forschung, wird jedoch die Lernumgebung nicht für den Lerner adaptiert, sondern der Lerner kann aktiv in seine Lernumgebung eingreifen und diese an seine Bedürfnisse anpassen. Unweigerlich werden damit die Aktivitäten und Interessen, die Handlungsund Interaktionsmöglichkeiten des Lernenden in den Fokus gestellt. PLE erscheinen besonders für das selbst gesteuerte oder auch informelle Lernen von Personen mit guten Internet-Kompetenzen attraktiv.

Abbildung 4 zeigt die Fülle von Inhalten und Methoden (Standards), die in einem PLE-Konzept berücksichtigt werden (können). Das PLE-Konzept ermöglicht die Integration von vorher getrennten Anwendungen in eine Umgebung. So können Lerner unterschiedliche Aktivitäten in anderen digitalen Umgebungen in ein PLE integrieren, zum Beispiel Diskussionen aus unterschiedlichen Communitys, Kontakte und Aktivitäten in sozialen Netzwerken, in denen Lerner oft mehrere Profile haben, individuelle Medienprodukte wie Bilder, Videos oder auch Musik, die auf externen Plattformen gespeichert werden, eigene Produkte (wie beispielsweise Tutorien), Nachrichten und Artikel aus unterschiedlichen Quellen oder Bookmarks aus Netzwerken.

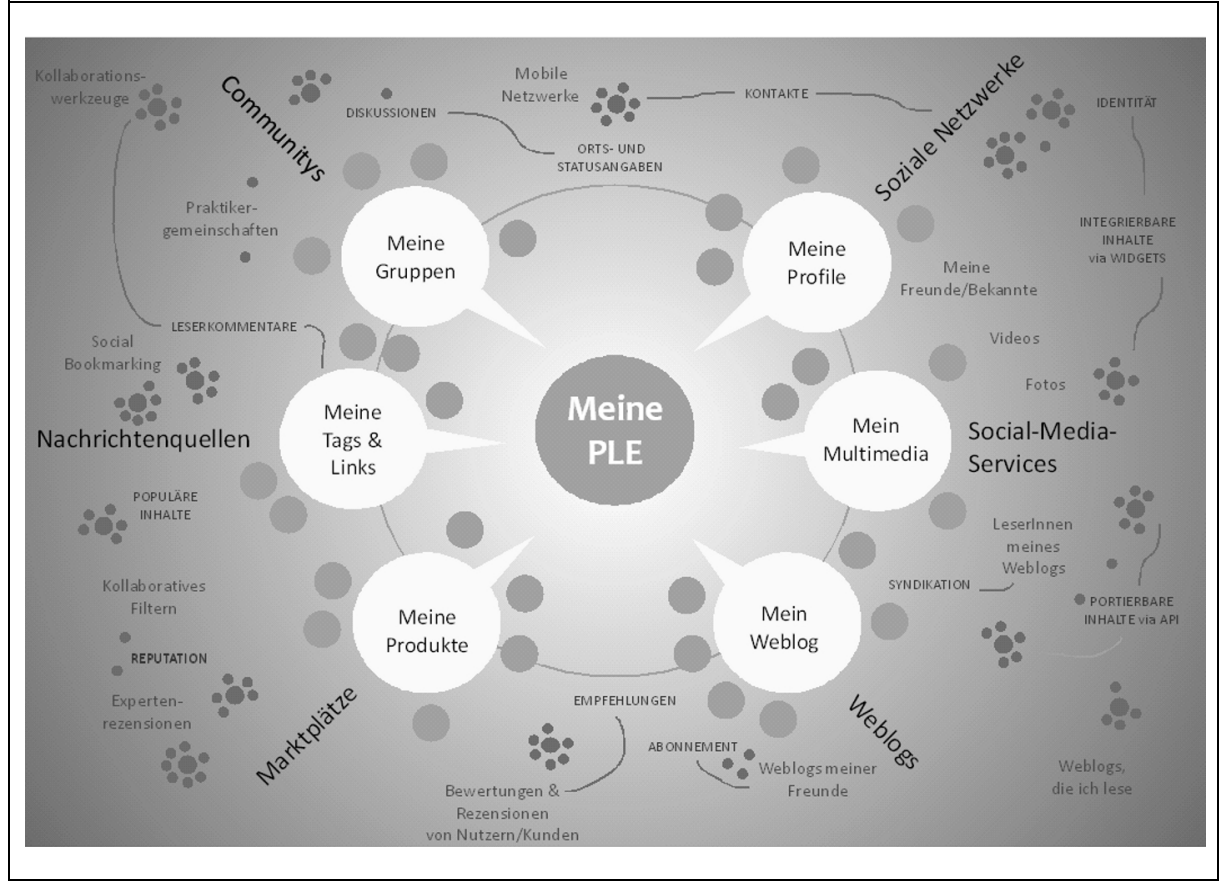

Abb. 4: Inhalte und Methoden, die im PLE-Konzept berücksichtigt werden sollten, nach einer Darstellung von TURNER (2008, von uns übersetzt und modifiziert)

Insbesondere reformpädagogische Ansätze stellen aktive Lernende in den Vordergrund ihrer pädagogischen Bemühungen, indem sie deren Selbststeuerung und Eigenaktivität ansprechen. Ebenso wird selbst organisiertes Lernen, das häufig auch informell und beiläufig am Arbeitsplatz oder bei der Ausübung eines Ehrenamts stattfindet, als wesentlicher Bestandteil des

Selbst organisiertes Lernen 
lebenslangen Lernens betrachtet. Insbesondere bei Erwachsenen, die bereits ihre Berufsaubildungen hinter sich haben, ist die Fähigkeit und Kompetenz zum selbst organisierten Lernen bedeutend für die weitere persönliche und berufliche Entwicklung: In der Regel werden nur ein Teil der Kompetenz- und Wissenserweiterungen durch die Teilnahme an Seminaren oder anderen Weiterbildungen unterstützt. Je mehr die Arbeit dabei mit oder am Computer oder im Internet ausgeführt wird, desto nahe liegender ist es, das selbst organisierte Lernen, das sonst beispielsweise durch das Lesen von Fachzeitschriften oder der Diskussion mit Experten erfolgte, auf das Medium Internet zu verlagern: Hier werden nicht nur Informationen recherchiert, sondern Wissen wird auch mit anderen kommuniziert und/oder gemeinsam entwickelt.

Selbst organisiertes Lernen kann als Aktivität betrachtet werden, bei der vorrangig die Individuen verantwortlich für ihre Planungen und Leistungen sowie für die Evaluation ihrer Lernaktivitäten sind, um ihre spezifischen Lernziele zu erreichen. Ein verwandtes Konzept wird »selbst gesteuertes Lernen « genannt. Malcolm Knowles beschreibt diesen Ansatz als Prozess »in dem Individuen die Initiative ergreifen, mit oder ohne Hilfe von anderen, um ihre Lernbedürfnisse $\mathrm{zu}$ diagnostizieren, Lernziele $\mathrm{zu}$ formulieren, menschliche und materielle Ressourcen für das Lernen zu identifizieren, angemessene Lernstrategien auszuwählen und umzusetzen« $(1975$, S. 18). Erziehungswissenschaftler ziehen häufig (sozial-) konstruktivistische Lerntheorien heran, wenn sie über lernerzentrierte Ansätze sprechen, die die Interessen der Lernenden und deren selbst gesteuerte Lernaktivitäten fokussieren: Ursprünglich ein philosophischer Ansatz, wird der Konstruktivismus häufig im Lernbereich genutzt und dazu adaptiert. Die konstruktivistische Perspektive auf das Lernen resultiert in Lernarrangements, die die eigene Konstruktion des Wissens der Lernenden besonders gut ermöglichen.

Anforderungen des institutionellen Lernmanagements wie Lernerverwaltung werden im PLE-Konzept hingegen nicht besonders berücksichtigt bzw. wird hier davon ausgegangen, dass diese Aufgaben, die eher im institutionellen Interesse als im individuellen liegen, von anderen externen Diensten übernommen werden. Dies hat unter anderem Implikationen für die Einsatzmöglichkeiten und die Rolle der Lernenden und Lehrenden, auf die wir später, nach der Darstellung einiger Realisierungen von PLE, noch ausführlich eingehen werden.

Passend zur Dynamik und Offenheit des PLE-Konzepts wird ein großer Teil der Diskussion über PLE wiederum in Weblogs geführt, auch wenn im letzten Jahr zunehmend traditionelle wissenschaftliche Beiträge auf Konferenzen und in internationalen Journalen veröffentlicht wurden (vgl. LIBER/JOHNSON 2008; Wild/Kalz/Palmer 2008).

\section{Realisierungen von PLE}

Wie im vorherigen Teil unseres Beitrags schon diskutiert, handelt es sich bei »Persönlichen Lernumgebungen « in erster Linie um ein technologisches Konzept. Im Folgenden möchten wir einige Formen von Anwendungen charakterisieren und beschreiben, die (auch) unter dem Stichwort »PLE« umgesetzt wurden. Wir haben dazu versucht, unterschiedliche Ansätze der Realisierung aus technologischer Perspektive zu identifizieren und zu unterscheiden. 
Generell kann man sagen, dass es PLE-Realisierungen gibt, die sich besonders im Aspekt der Einbindung externer Dienste und Web-Services unterscheiden. Während es Ansätze gibt, bei denen nur sehr eingeschränkt die Anpassung der Umgebung durch den Lernenden ermöglicht wird, gibt es auf der anderen Seite Realisierungen, bei denen ausschließlich externe Dienste und Web-Services eingebunden werden und der Lernende volle Kontrolle über die Bestandteile seiner Lernumgebung hat. WILD, MöDriTsCHER UND Sigurdarson (2008) nennen diesen Aspekt der Komposition der eigenen Lernumgebung »Lernumgebungsdesign «. Im Folgenden wollen wir drei beispielhafte Umsetzungen beschreiben, die sehr gut als Prototypen dienen können. Zwischen diesen Prototypen existieren eine Vielzahl weiterer Umsetzungsmöglichkeiten des PLE-Konzeptes.

\subsection{Service-Integration in vorhandene Lernumgebungen}

Eine leichte Öffnung hin zur Anpassbarkeit von Lernumgebungen durch den Lerner wird u. a. durch die Möglichkeit der Einbindung von RSS-Feeds in klassische Lernumgebungen wie z. B. LMS realisiert. Sowohl einige Open-SourceProjekte als auch kommerzielle Systeme bieten mittlerweile die Möglichkeit der Einbindung von externen Services. Hier können sich Lernende also neben den klassischen Funktionalitäten eines LMS ihren individuellen Bereich schaffen, in dem sie einerseits wieder Informationen und Dienste sowohl auf individueller als auch auf Gruppenebene integrieren und ggf. miteinander teilen. So ist es z. B. in verschiedenen Systemen möglich, einen gemeinsamen externen Gruppenkalender in ein LMS zu integrieren, RSS-Feeds in das Interface einzubauen oder externe Medien im LMS einzubinden. Hier wird das PLE-Konzept auf eine vorhandene, vorstrukturierte Lernumgebung angewendet, jedoch ist die Eingriffsmöglichkeit des Lerners begrenzt.

\subsection{Integrierte Oberflächen}

Zur dieser Gruppe gehören zum Beispiel »persönliche Portale« oder »persönliche Startseiten «, die es dem Benutzer ermöglichen, verschiedene Informationsquellen und Dienste in einem Interface zu bündeln, und somit eine Art individuelles »Cockpit « für die verschiedenen Anwendungen und Informationsquellen des Lerners bieten. Dies kann zu Zeitersparnissen führen, weil Benutzer alle Informationen im Blick haben können, und auch zu einer effizienteren Informationsnutzung führen, da durch die Zusammenführung mehrere Informationsquellen kontrollierbar werden. Diese persönlichen Startseiten werden als kostenlose Dienste von z. B. Netvibes oder Google angeboten. Hier kann man beispielsweise durch sogenannte »Widgets « externe Services in seinem persönlichen Portal einbinden. So können bei Google registrierte Benutzer in ihrem persönlichen Portal mit dem Namen »I-Google « Widgets einbinden, die die Recherche in einem externen Sprachlexikon (wie Leo.org) oder das Lesen der neuesten Sprach-Lektion ermöglichen, ohne die I-Google-Seiten zu verlassen. Der Schutz der persönlichen Daten, beispielsweise der bevorzugten Weblogs oder Suchbegriffe ist folglich nur eingeschränkt vorhanden, soweit man solche Angebote nutzt. Wer potenzielle Eingriffe vermeiden möchte, aber dennoch trotzdem vergleichsweise flexibel, d. h. auch im Internetcafe auf sein PLE zugreifen möchte, kann beispielsweise ein persönliches Content-Management-System nutzen und als PLE

Handbuch E-Learning 27. Erg.-Lfg. Januar 2009 
ausbauen, sofern die Voraussetzungen dazu (z. B. eigener Webserver) gegeben sind. Daneben gibt es Anwendungen, die externe Services integrieren, die man installieren muss. Dazu gehören beispielsweise Plug-Ins, also kleine $\mathrm{Zu}$ satzprogramme für den Web-Browser, oder auch der Browser »Flock «, der sich auf die Einbindung unterschiedlichster Social-Software-Services spezialisiert hat und damit auch als PLE dienen kann. Ein Vorteil einer solchen Lösung ist, dass man Zugangsdaten zu anderen Services, wie zum Beispiel dem persönlichen Wiki-Space, nicht kommerziellen Anbietern (wie beim Beispiel oben: Google) weitergibt, sondern diese Zugangsdaten lokal auf dem eigenen Rechner gespeichert sind. Der Nachteil eines solchen Tools ist hingegen, dass man es nicht von anderen Rechnern aus nutzen kann, da ja eben eine lokale Installation notwendig ist.

\subsection{PLE als Framework}

Schließlich gibt es noch eine dritte Art von PLE-Projekten, die sich das Rahmenwerk, das Framework, von PLE vornehmen: Mit Hilfe eines Frameworks kann man eigene Anwendungen, z. B. die oben genannten Anwendungen, zusammenbauen. Frameworks fassen eine Menge aufeinander abgestimmter Dienste zusammen und ermöglichen es, darauf aufbauend Anwendungen zu entwickeln. In einer Lernumgebung würde für die Benutzerverwaltung z. B. auf externe Dienste wie OpenID zurückgegriffen werden, um den Zugang zu den Inhalten zu ermöglichen. Die Inhalte könnten aus Lernobjekt-Repositorien gespeist werden, die zum individuellen Profil des Lerners passen. Die Arbeit mit Frameworks ist jedoch nur sehr kompetenten Lernern vorbehalten. Infrastrukturen zum Entwickeln von PLE, sogenannte »Frameworks « (Rahmenwerke), werden zurzeit in unterschiedlichen von der Europäischen Kommission ko-finanzierten Projekten wie z. B. TENCompetence, iCamp und MATURE entwickelt.

Neben diesen prototypischen Umsetzungen des PLE-Konzeptes werden diese zum Teil auch in vorhandenen Web-Anwendungen wie Weblogs oder Wikis realisiert, die - je nach System - mehr oder weniger dazu geeignet sind, vom Endanwender nach eigenen Bedürfnissen angepasst und verändert zu werden.

\section{$5 \quad$ Das Konzept der PLE im Vergleich mit traditionellen technologischen Lehr- und Lernkonzepten}

Im Unterschied zu den gerade erwähnten didaktischen Konzepten gibt es auch weitere technologische Konzepte des Lehrens und Lernens. Solche technologischen Konzepte sind nicht zwangsläufig mit bestimmten didaktischen Vorgehensweisen oder pädagogischen Ansätzen verbunden, dennoch führen sie letztlich zu bestimmten Lern-Settings.

\subsection{Vergleich mit vorhandenen technologischen Konzepten}

Das Konzept von PLE gliedert sich in eine ganze Reihe von technologischen Lehr- und Lernkonzepten ein, die für das Lernen mit dem Computer und dem Internet entwickelt wurden. Im Unterschied zu didaktischen Konzepten 
zeichnen sie sich dadurch aus, dass es bei ihnen primär um die technologische Realisierung geht und weniger um die Beschreibung oder Realisierungen eines didaktischen Ansatzes, wie es beispielsweise im Bereich des Lernens mit dem Internet die E-Portfolio-Methode oder der Ansatz von Webquests darstellt.

Computer Based Trainings (CBT) bzw. Web Based Trainings (WBT) verfolgten den Ansatz, Inhalte von Lehrtexten und -bücher multimedial auszuschmücken, beispielsweise durch Einbindung von Videos und Bildern, oder sie durch kleinere interaktive Aktionen wie Mehrfachauswahl-Tests attraktiv zu machen. Eine Interaktion mit anderen Lernenden war hierbei nicht vorgesehen, in der Regel müssen dabei auch längere Sequenzen »durchgeklickt « werden.

Intelligente Tutor Systeme (ITS) wiederum basieren auf der Vorstellung, dass ein intelligentes System in der Lage ist, kleine Lerneinheiten und Sequenzen für einzelne Lerner individualisiert, z. B. abhängig von seinem Lernertyp, zu arrangieren und darzustellen.

- Lern-Management-Systeme schließlich unterstützen das Management von Lerninhalten und Aktivitäten aus der Perspektive einer Bildungsinstitution. Das Lernen im Klassenzimmer wird dabei imitiert, wobei die Rollenverteilung traditionell ist: Der Lehrer entwickelt und bietet Lerninhalte an.

Vergleicht man diese bestehenden Konzepte des computer- und internetgestützten Lernens mit dem Konzept der PLE fallen die Besonderheiten schnell ins Auge: Ein wenig überzeichnet, und sicher nicht für jede Realisierung zutreffend, sollen die folgenden beiden Abbildungen hier als Illustration dienen.

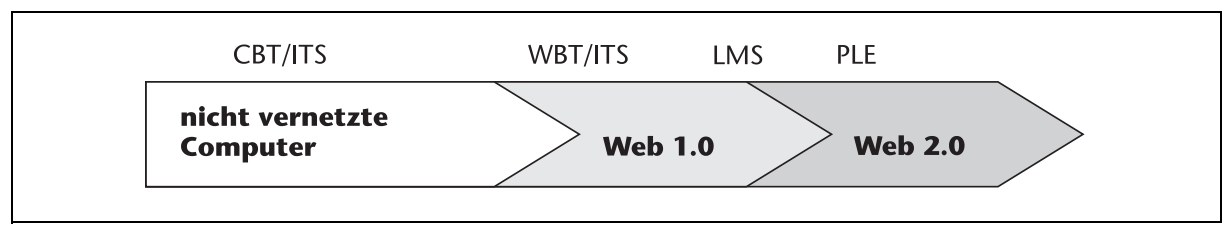

Abb. 5: Ausgewählte technologische Lehr-/Lernkonzepte und ihre technologische Heimat

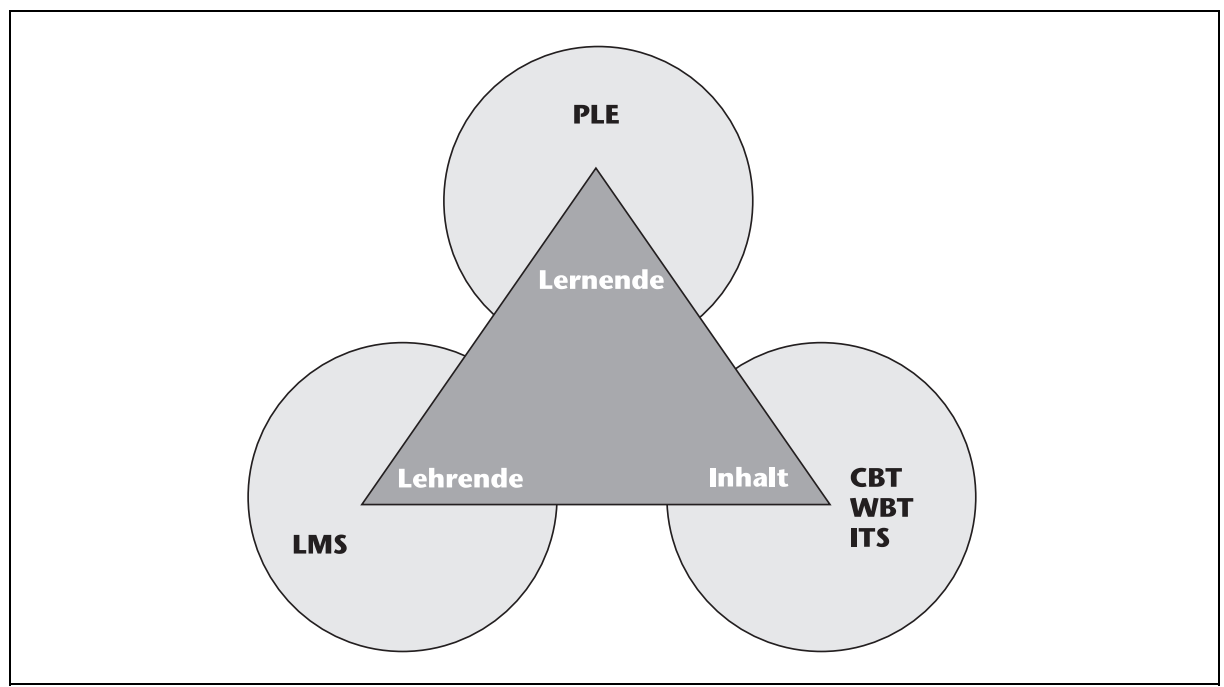

Abb. 6: Ausgewählte technologische Lehr-/Lernkonzepte und ihre Perspektive auf die Komponenten des didaktischen Dreiecks 
Abbildung 5 zeigt die unterschiedlichen Technologien, auf die die Konzepte zurückgreifen: Während Intelligente Tutoren Systeme (ITS) und Computer Based Trainings (CBT) dazu entwickelt wurden, auf zunächst nicht vernetzten Computern zu laufen, wurden durch die Einführung von Intra- und Internet auch Web Based Trainings (WBT) und web-basierte ITS-Anwendungen möglich. Aktuelle Realisierungen von Lern-Management-Systemen (LMS) agieren an der Schnittstelle vom Web 1.0 und Web 2.0. Mit diesen Versionsnummern wird der Wandel des Internets bezeichnet, der unter anderem auch maßgeblich durch die neuen Social-Software-Anwendungen bestimmt ist. Technologische Grundlage des PLE-Konzepts sind schließlich genau diese ganz neuen Entwicklungen.

Abbildung 6 zeigt, - sehr zugespitzt - wie sich die Perspektive auf das Lernen in den ausgewählten technologischen Konzepten unterscheidet, und nutzt dazu die traditionellen Größen des didaktischen Dreiecks, nämlich Lernende, Lehrende und Inhalt. Während das PLE-Konzept deutlich den Lernenden und seine Bedürfnisse im Fokus hat, dreht es sich bei den technologischen Konzepten CBT, WBT und ITS vor allem um die Inhalte und ihre Darstellung und Vernetzung. In einem ITS evaluiert das System die Lernenden und ihr Verhalten und arrangiert auf dieser Grundlage weitere Lerninhalte, dem Lernenden werden im Unterschied zur PLE keine Möglichkeiten für eigenständige Entscheidungen eingeräumt. Das Konzept des LMS hat zunächst einmal vor allem die Bedürfnisse der Lehrenden und der Institution im Blick, indem es eben viele administrative Lösungen bei der Verwaltung bietet.

\subsection{Vergleich mit Lern-Management-Systemen}

Das wohl am weitesten verbreitete technologische Konzept der Lern-Management-Systeme bestimmt die E-Learning-Realität, insbesondere in formalen Settings im Bereich von Hochschulen und auch von betrieblichen bzw. beruflichen Weiterbildungen. Im Vergleich dazu haben IST nur in bestimmten Disziplinen und eingeschränkt praktische Relevanz. Da erscheint es nahe liegend, PLE im Detail mit LMS zu vergleichen und zu betrachten, welche grundlegenden Veränderungen für den erfolgreichen Einsatz von PLE notwendig bzw. auch zu erwarten wären. Anzumerken bleibt, dass derzeit nicht absehbar ist, dass PLE, zumal in formalen Settings, überhaupt in der Lage wären, eine echte Konkurrenz zu LMS darzustellen - eben weil es einen ganz anderen Ansatz verfolgt: Das Lernen in PLE erfolgt eben nicht fremdbestimmt und kann auch nicht von anderen eingesehen oder administrativ verwaltet werden. Die folgende Tabelle gibt einen Überblick über sieben wichtige Aspekte des potenziell denkbaren, aber real derzeit wohl nur selten durch Institutionen initiierten Wechsels von LMS zum PLE-Konzept (vgl. SCHAFFERT/HILZENSAUER 2008). 


\begin{tabular}{|c|c|c|c|c|}
\hline & & LMS & PLE & $\begin{array}{l}\text { Heraus- } \\
\text { forderungen \& } \\
\text { Veränderungen }\end{array}$ \\
\hline 1 & $\begin{array}{l}\text { Rolle der } \\
\text { Lernenden }\end{array}$ & $\begin{array}{l}\text { Lernende als } \\
\text { Konsumenten } \\
\text { vordefinierter } \\
\text { Lernmaterialien, } \\
\text { abhängig von } \\
\text { der »Kreativität« } \\
\text { der Lehrenden }\end{array}$ & $\begin{array}{l}\text { Aktive, selbst } \\
\text { gesteuerte } \\
\text { Produzenten von } \\
\text { Inhalten }\end{array}$ & $\begin{array}{l}\text { Der Wechsel vom } \\
\text { Konsumenten zum } \\
\text { »Prosumenten«, } \\
\text { Selbstorganisation } \\
\text { ist möglich UND } \\
\text { notwendig }\end{array}$ \\
\hline 2 & $\begin{array}{l}\text { Personali- } \\
\text { sierung }\end{array}$ & $\begin{array}{l}\ldots . \text { ist ein Arrange- } \\
\text { ment von Übungs- } \\
\text { aufgaben und } \\
\text { Lernmaterialien } \\
\text { gemäß einem } \\
\text { vorgeschlagenen } \\
\text { oder vordefinier- } \\
\text { ten Profil der } \\
\text { Lernenden. }\end{array}$ & $\begin{array}{l}\text {... bedeutet, } \\
\text { Information über } \\
\text { Lernmöglichkeiten } \\
\text { und Inhalten von } \\
\text { Community-Mit- } \\
\text { gliedern und Lern- } \\
\text { unterstützungen } \\
\text { zu erhalten, die zu } \\
\text { den Interessen der } \\
\text { Lernenden passen } \\
\text { (z. B. via } \\
\text { RSS-Feeds). }\end{array}$ & $\begin{array}{l}\text { Kompetenz im } \\
\text { Umgang mit } \\
\text { unterschiedlichen } \\
\text { Werkzeugen und } \\
\text { Selbstorganisation } \\
\text { sind notwendig. }\end{array}$ \\
\hline 3 & Lerninhalte & $\begin{array}{l}\text { Werden von } \\
\text { Domänen- } \\
\text { experten, speziel- } \\
\text { len Autoren, } \\
\text { Tutorlnnen oder } \\
\text { Lehrenden } \\
\text { entwickelt. }\end{array}$ & $\begin{array}{l}\text { Der unendliche } \\
\text { Bazar von } \\
\text { Lerninhalten, } \\
\text { Lernmöglichkeiten } \\
\text { und -Services }\end{array}$ & $\begin{array}{l}\text { Notwendige } \\
\text { Kompetenzen, } \\
\text { passende Quellen } \\
\text { zu suchen, zu } \\
\text { finden und zu } \\
\text { benutzen } \\
\text { (z. B. Weblogs) }\end{array}$ \\
\hline 4 & $\begin{array}{l}\text { Soziale } \\
\text { Beteiligung }\end{array}$ & $\begin{array}{l}\text { Eingeschränkte } \\
\text { Nutzung von } \\
\text { Gruppenarbeit, } \\
\text { der Fokus liegt } \\
\text { dabei auf } \\
\text { geschlossenen } \\
\text { Lernergruppen } \\
\text { (z. B. im LMS); } \\
\text { Kollaboration und } \\
\text { Austausch stehen } \\
\text { nicht im Fokus. }\end{array}$ & $\begin{array}{l}\text { Die Community } \\
\text { und die soziale } \\
\text { Beteiligung (auch } \\
\text { bei mehreren } \\
\text { Communitys) ist } \\
\text { der Schlüssel für } \\
\text { den Lernprozess } \\
\text { und Empfehlun- } \\
\text { gen für Lern- } \\
\text { möglichkeiten }\end{array}$ & $\begin{array}{l}\text { Community und } \\
\text { Kollaboration als } \\
\text { zentrale Lern- } \\
\text { möglichkeiten }\end{array}$ \\
\hline 5 & $\begin{array}{l}\text { Eigentümer- } \\
\text { schaft }\end{array}$ & $\begin{array}{l}\text { Inhalte gehören } \\
\text { im Allgemeinen } \\
\text { den Bildungs- } \\
\text { einrichtungen } \\
\text { oder den Lernen- } \\
\text { den; aus tech- } \\
\text { nischen Gründen } \\
\text { können letzte } \\
\text { Eigentümer- } \\
\text { schaften nicht } \\
\text { immer auch } \\
\text { realisiert werden. }\end{array}$ & $\begin{array}{l}\text { Inhalte sind in } \\
\text { mehreren web- } \\
\text { basierten Werk- } \\
\text { zeugen organi- } \\
\text { siert, die Eigen- } \\
\text { tümerschaft wird } \\
\text { von den Lernen- } \\
\text { den selbst oder } \\
\text { (kommerziellen) } \\
\text { Service-Anbietern } \\
\text { kontrolliert. }\end{array}$ & $\begin{array}{l}\text { Aufmerksamkeit } \\
\text { für persönliche } \\
\text { Daten ist } \\
\text { notwendig. }\end{array}$ \\
\hline
\end{tabular}




\begin{tabular}{|c|l|l|l|l|}
\hline & & LMS & PLE & $\begin{array}{l}\text { Heraus- } \\
\text { forderungen \& } \\
\text { Veränderungen }\end{array}$ \\
\hline 6 & $\begin{array}{l}\text { Lern- und } \\
\text { Organisations- } \\
\text { kultur }\end{array}$ & $\begin{array}{l}\text { Imitation des } \\
\text { Lernens im } \\
\text { Klassenzimmer, } \\
\text { Kurs- und Lehrer- } \\
\text { orientierte Merk- } \\
\text { male }\end{array}$ & $\begin{array}{l}\text { Der selbst } \\
\text { organisierte } \\
\text { Lernende steht } \\
\text { im Fokus. }\end{array}$ & $\begin{array}{l}\text { Wechsel der } \\
\text { Lernkultur und der } \\
\text { Perspektive, ein } \\
\text { Wechsel zu Selbst- } \\
\text { organisation und } \\
\text { Selbstbestimmung }\end{array}$ \\
\hline 7 & $\begin{array}{l}\text { Technologische } \\
\text { Aspekte }\end{array}$ & $\begin{array}{l}\text { Klassische Lern- } \\
\text { inhalte benötigen } \\
\text { Interoperabilität } \\
\text { von LMS und } \\
\text { Datenbanken. }\end{array}$ & $\begin{array}{l}\text { Social-Software- } \\
\text { Anwendungen } \\
\text { und Aggregation } \\
\text { einer Vielzahl } \\
\text { von Quellen }\end{array}$ & $\begin{array}{l}\text { Interoperabilität } \\
\text { zwischen LMS und } \\
\text { Social Software } \\
\text { wird notwendig. }\end{array}$ \\
\hline
\end{tabular}

Abb. 7: Vergleich der Konzepte von Lern-Management-System und Persönlicher Lernumgebung und daraus folgende Veränderungen nach SCHAFFERT und HILZENSAUER (2008)

Lernende als "Prosumenten«

Vor- und Nachteile
Analog zu den Entwicklungen der ersten Jahre im World Wide Web, bei denen die Mehrheit der Internetnutzer als Leser von Webseiten, Nutzer von Informationen oder Kunden bei Online-Shops vorrangig als Konsumenten in Erscheinung traten, sind auch Lernende bis vor wenigen Jahren überwiegend passiv gewesen: Lerninhalte wurden in herkömmlichen Konzepten in der Regel von Experten erstellt und entwickelt. Lernende sind dort eben auch vorrangig »Konsumenten «, indem sie surfen, lesen und Materialien nutzen (z. B. Tests oder Lernspiele), aber keine Möglichkeit haben, sich aktiv an der Entwicklung von (Lern-)Inhalten zu beteiligen. Mit den Anwendungen des Web 2.0 wurde es möglich, vergleichsweise einfach Inhalte zu erstellen, z. B. via Weblogs und Wikis. Auch Lernende wurden, beispielsweise indem sie in Wikis kollaborativ Projektarbeiten entwickelten, zu aktiven Beitragenden, zu »Prosumenten «. Im Zentrum des Ansatzes von PLE steht eben diese aktive Partizipation bei der (kollaborativen) Entwicklung von (Lern-)Materialien, z. B. durch Weblog-Artikel, Beiträge auf Wiki-Seiten, Teilnahme in Diskussionsforen oder das Kommentieren von Weblog-Beiträgen anderer.

Während das LMS-Konzept die möglichen Rollen der Lerner auf die Möglichkeiten des Systems und die Kreativität der Lehrenden einschränkt - Lerner haben hier in der Regel nur eingeschränkte Rechte, Inhalte zu entwickeln fokussiert das PLE-Konzept den aktiven, selbst gesteuerten Entwickler von Inhalten. Die Selbstorganisation des Lernens wird oft als Basis für die Kompetenzentwicklung und die Fähigkeit zu lebenslangen Lernen betrachtet, die bereits in den Schulen entwickelt werden sollen. Problematisch ist jedoch, dass die Fähigkeit zur Selbstorganisation nicht bei jedem Lernenden in gleich hohem Ausmaß vorausgesetzt werden kann. Auch Erwachsene benötigen häufig Unterstützung, Tutoring oder auch Weiterbildungen, um das eigene Lernen selbst organisieren zu können. Nicht nur die Notwendigkeit eines (eigenen) Computers und Internetzugangs sowie die erforderlichen Kompetenzen im Umgang damit schließen zudem bestimmte Personengruppen aus: Das »selbst organisierte Lernen « als Konzept passt vor allem zu Mittelschicht-Milieus und schließt damit Zielgruppen bzw. Milieus aus, die lehrerzentrierte Ansätze bevorzugen (siehe auch BREMER/BITTLINGMAYER 2008). 
In Lern-Management-Systemen sind die Möglichkeiten der Personalisierung nur eingeschränkt. Beispielsweise hat der Nutzer Zugang zu den für sie/ihn freigegebenen Kursen oder Klassen. Eine weitere Differenzierung der Instruktion oder der Lernmaterialien oder sogar Personalisierung ist abhängig von den Lehrenden und deren kreativen Ideen, aber nur selten in LMS umgesetzt. Personalisierung ist im E-Learning traditionell mit intelligenten Tutorsystemen verknüpft; z. B. verfolgt der Ansatz des »Instructional Design« die Idee, das Lernen zu unterstützen, indem man relativ kleine Lerneinheiten entwickelt und anbietet. Durch implementierte intelligente Tutoren, werden Lernwege vorgegeben oder Darstellungen gewählt, die den Lernerprofilen - Lernertypen oder Vorwissen - entsprechen. Für das selbst organisierte Lernen sind solche Formen der Personalisierung nur eingeschränkt brauchbar, weil sie die Möglichkeiten einschränken, selbst Lernschritte zu strukturieren und zu organisieren. Im PLE-Konzept bedeutet »Personalisierung «, die Aktivitäten und Möglichkeiten, die Strukturen, Werkzeuge und (externe) aggregierte Materialien, die Handhabung und das Design selbst bestimmen zu können. Personalisierung heißt hier, Informationen über die Lernmöglichkeiten und -inhalte aus vielen Communitys und Services zu erhalten, die zu den Interessen des Lerners passen. Diese Quellen werden durch die Lernenden selbst gewählt.

Natürlich stellt die Selbstorganisation der Lernenden nicht nur eine große Chance für die Lernenden dar, sondern erfordert es auch, über die Voraussetzungen der Lernenden nachzudenken: über ihre Kompetenzen und notwendiges Vorwissen in Bezug auf die Benutzung von Social-Software-Anwendungen, ihre unterschiedlichen Lernstrategien und ihre Medienkompetenz, auch im Umgang mit und bei der Vielzahl von Quellen und Ressourcen die (potenziell) zur Verfügung stehen.

In LMS ist die Rolle der Teilnehmer klar definiert und beschrieben und legt fest, in welcher Weise man im System interagieren kann: Die Inhalte von LMS werden von Lehrenden entwickelt, die hauptsächlich Experten einer bestimmten Domäne sind. Im Web 2.0 und auch im PLE-Konzept können dagegen auch Externe, sogar die Lernenden selbst, an der Entwicklung von Lernressourcen beteiligt sein. Die PLE enthält eben nicht nur Lerninhalte, die von Experten oder Lehrern entwickelt werden; sondern auch Freunde, Kollegen, Menschen mit ähnlichen Interessen oder Personen, die man nicht persönlich kennt, entwickeln einen Bazar von kostenlosen Lernmöglichkeiten. PLE profitieren von der Entwicklung, dem Angebot und dem Gebrauch von Offenen Bildungsressourcen (Open Educational Resources). »Offen« bedeutet dabei, dass die Inhalte, inklusive Metadaten, kostenlos und frei lizenziert angeboten werden und dass die Nutzung und im besten Falle auch die Veränderung und Neukombination erlaubt ist. Im weiteren Sinne bzw. im besten Falle sollten mit OER im Sinne ihrer Promotoren auch Methoden offenen Unterrichts und Open Source Software zum Einsatz gelangen (vgl. GESER 2007).

Nichtsdestotrotz führt der Wechsel von Inhalten, die von Experten und Lehrern entwickelt wurden, zu den Möglichkeiten und Herausforderungen des Bazars an Lernmöglichkeiten auch zu der Notwendigkeit fortgeschrittener Selbstorganisation und Webrecherche - in anderen Worten: Es bedarf medienkompetenter Lerner. Auch die nachwachsenden Generationen, sind entgegen aller Erwartungen an so genannten »digital natives « auch nicht medienkompetenter als die »digital immigrants«, Medienkompetenz ist also
Neue Bedeutung von Personalisierung

Bazar der Lernmöglichkeiten 
nicht vorauszusetzen (SCHULMEISTER 2008). Technologisch ist die Integration der unterschiedlichen Lern-Settings, unterschiedlicher Lernergruppen und unterschiedlicher Ressourcen eine Herausforderung. Wir denken jedoch, dass diese Integration in PLE flexibler und nahtloser gelingen kann als in LMS.

Lern-Netzwerke

Eigentümerschaft und Datenschutz
Wir behaupten nicht, dass es unmöglich ist, innerhalb eines LMS eine intensive Kollaboration von Lernenden aufzubauen und zu pflegen, aber dies gehört nicht zur Kernidee dieses technologischen Konzepts. LMS ermöglichen zwar, dass man Diskussionsforen hinzufügt, manchmal auch kollaborative (Wiki-) Räume, aber Kollaboration und sozialer Austausch stehen nicht im Fokus des LMS-Konzepts. Im PLE-Konzept ist es dagegen augenscheinlich, dass die Verbindung zu einer oder mehreren Lern-Communitys der Schlüssel zum Lernen ist. Auch in technologischer Hinsicht benötigt das PLE-Konzept Communitys bzw. Lernnetzwerke und baut darauf auf: Die »Anderen « werden als Beitragende, Ko-Handelnde oder auch als Produzenten von Meta-Informationen gebraucht; mit letzteren können beispielsweise neue oder empfehlenswerte Lernressourcen angezeigt oder Metadaten zu Lernressourcen erstellt werden.

Auch die Eigentümerschaft und der Datenschutz sind wichtige Faktoren, werden jedoch in der Regel kaum beachtet. Aus unserer Sicht sollten sie jedoch eine größere Rolle für die Umsetzungen von E-Learning spielen. Bei LMS und PLE unterscheiden sich die Rechte der Nutzer augenscheinlich und rufen dabei ganz unterschiedliche Probleme hervor: Die Daten der Lerner sind in LMS häufig nicht leicht zugänglich und können selten von den Lernenden aus den LMS gelöscht bzw. woanders zufriedenstellend portiert werden. Selbst als Eigentümer ihrer Daten und Inhalte haben Lernende faktisch nur eingeschränkte Möglichkeiten und ihre Daten stehen unter der Kontrolle der Bildungseinrichtung oder Organisation. Das PLE-Konzept und seine Offenheit verfolgen einen ganz anderen Ansatz, auch wenn die Realisierungen wie »Elgg « zum Teil auch zugangsbeschränkte Bereiche anbieten. Fast alle Daten und erhältlichen Informationen sind nahezu grenzenlos für andere einsehbar. Weil Nutzer in der Regel keine eigenen Webserver nutzen, sondern kostenlose Web-Services und -Werkzeuge, sind häufig ihre persönlichen Daten und auch ihre entwickelten Inhalte durch die Service-Anbieter geschützt. Beides führt zu der Notwendigkeit, Lernende auf diese Herausforderungen hinzuweisen. Hinzu kommt noch ein technischer Aspekt: Beim Nutzen externer Services sollte man nicht vergessen, Kopien und Sicherungen der Daten anzulegen.

Lernkultur Wie bereits erwähnt, unterstützen LMS traditionelle Wege, das Lernen und die Bildung zu organisieren: in Unterrichtsstunden, Kursen, Klassen, aus Sicht der Administration und mit einem Lehrer als Experten. Das PLE-Konzept konzentriert sich auf aktive Lernende, die für das eigene Lernen verantwortlich sind und die Möglichkeit haben, ihre eigene Lernumgebung zu gestalten. Die Nutzung von PLE oder neuen Formen des Lernens mit Social Software im Allgemeinen kann als selbst bestimmt, dezentralisiert und dynamisch beschrieben werden; Lernende entwickeln dabei an den Lernressourcen mit und sind so nicht nur Konsumenten, sondern auch »Prosumenten «. Allerdings bringen Bildungseinrichtungen wie auch berufliches Lernen oder das Lernen am Arbeitsplatz nicht automatisch die Vorraussetzungen mit, die für diese Art der Kompetenzentwicklung gegeben sein müssen. Untersuchungen und Evaluationen bestätigen, dass Veränderungen der Lernkultur 
einer Einrichtung und ein gewisser Anteil an Offenheit in der gesamten Einrichtung einen guten Nährboden für erfolgreiches Lernen in Unternehmen bilden können.

Zurzeit beschäftigen sich eine Reihe technologischer Entwicklungen mit dem Austausch von Objekten in unterschiedlichen Datenbanken, z. B. unterschiedlicher Institute. Weil es immer mehr frei erhältliche Ressourcen gibt, ist es notwendig, diese Materialien verknüpfen zu können und präzise Metadaten anzubieten. Daraus folgt, um den Standards zu genügen, dass die Lerninhalte und das Lern-Management-System gewährleisten müssen, dass die zu verknüpfenden Ressourcen auch kompatibel sind. Da LMS in hierarchischen Strukturen organisiert werden, bei denen Autoren (die sogenannten Domänenexperten) entscheiden, zu welchen externen Quellen sie verlinken wollen oder was sie aggregieren wollen, liegt die Herausforderung darin, ein flexibles und dynamisches Interface aufzusetzen, mit dem diese externen Quellen verwaltet werden können. Mit Blick auf die Personalisierung erscheint es besonders schwierig, wenn Lehrende Materialien zur Verfügung stellen wollen, die ganz unterschiedliche Nutzer in unterschiedlichen Phasen des Lernens unterstützen sollen.

PLE bauen Social-Software-Anwendungen auf und benötigen daher einen Kanal für den Austausch von Informationen. Im Gegensatz zu den existierenden E-Learning-Standards (LOM, SCORM, IMS-LD) liegt der Bedarf beim Datenaustausch eher bei offenen Standards wie RSS, XML oder RPC.

Es liegt nahe, die Vorteile der Social-Software-Tools für das selbst organisierte Lernen auch in LMS zu nutzen. Es gibt mehrere Projekte, die versuchen, das Konzept von LMS mit den Möglichkeiten von PLE zu kombinieren: Der Nutzen von Social Software für die individuelle Kompetenzentwicklung und das Lernen führt zu Fragen bezüglich der Möglichkeiten, Social Software in existierende Stand-Alone-Systeme (wie LMS) einzubauen oder diese davon getrennt als verteilte Anwendungen zu nutzen (DAALSGARD 2006). Wenn sich LMS für Social Software öffnen, bedeutet das nicht nur, dass eine ganze Reihe von Lernressourcen nutzbar werden, sondern auch anderes Expertenwissen, das vorher in geschlossenen Umgebungen abgelegt wurde und nun durch Social Software externalisiert wird. Allerdings zeigt sich, dass es für die Verknüpfung weitestgehend getrennter Welten - das Web als offene Umgebung und das LMS als geschlossenes System, in dem Interoperabilität durch Standards abgesichert wird - Erkundungen dazu notwendig macht, wie Interoperabilität auch mit anderen als den derzeit implementierten Standards zu gewährleisten ist. Es zeigt sich, dass der Einbau von Social-Software-Anwendungen in existierende Lern-Management-Systeme eine ganze Reihe neuer Fragen und Herausforderungen aufwirft.

\section{Verwandte (didaktische) Methoden und Ansätze}

Das technologische Konzept »PLE« ist mit einigen anderen (didaktischen) Methoden und Ansätzen, die die Selbstorganisation von Individuen in den Fokus stellen, verwandt bzw. kann diese zumindest zum Teil unterstützen. Beispielhaft verweisen wir im Folgenden auf das persönliche (webbasierte) Wissensmanagement, auf die E-Portfolio-Methode sowie auf das Lernen in »Communities of Practice $\ll$.
Technologische Herausforderungen

Interoperabilität 


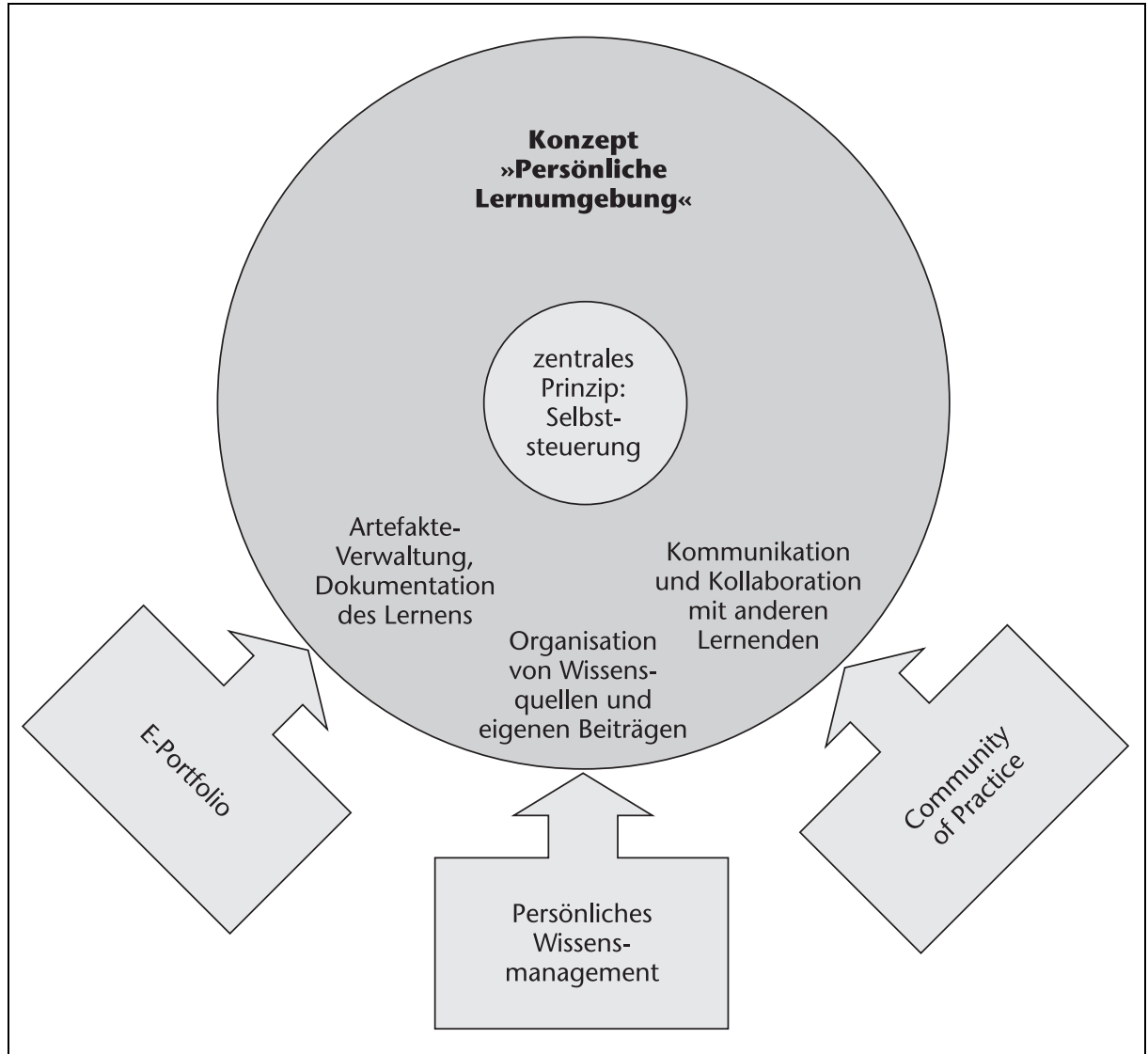

Abb. 8: Überschneidungen von drei ausgewählten (didaktischen) Methoden mit dem technologischen Konzept von PLE

Abbildung 8 zeigt, wo diese drei Ansätze Kompatibilität mit dem Konzept von PLE aufweisen, die in der Praxis durchaus vereinbare Zielvorstellungen darstellen könnten, nicht zuletzt deshalb, weil sie alle die Selbststeuerung von Lernenden bzw. von Lerngemeinschaften in den Fokus stellen. Dennoch geben wir auch Hinweise darauf, welche Besonderheiten die Methoden und das aktuelle Konzept von PLE bisher haben, die keine Übereinstimmungen aufweisen.

\subsection{PLE und persönliches Wissensmanagement}

Wer an Wissensmanagement denkt, assoziiert damit wohl zunächst Ansätze dahingehend, wie in Unternehmen oder anderen Organisationen mit Wissen umgegangen wird, um einen effektiven Austausch und Dokumentation zu ermöglichen. Das »persönliche Wissensmanagement « umfasst hingegen die individuellen Tätigkeiten, das eigene Wissen und externe Quellen bestmöglich zu dokumentieren und zu organisieren. Für Gabi ReINMANN ist persönliches Wissensmanagement daher auch ein genuin pädagogisch-psychologisches Thema: »Wissen aus der Umwelt aufnehmen, es auswählen und verstehen, neues Wissen entwickeln, es mit anderen teilen oder bei sich bewahren - all das sind mentale Prozesse des Menschen, die sich in sichtbarem oder weniger sichtbarem Handeln niederschlagen.«(2008a, S. 1). 
Aus dieser Perspektive ist das persönliches Wissensmanagement eng mit dem Lernen verknüpft: »Ein Personal Learning Environment (PLE) ist konzeptionell nichts anderes als die persönliche Wissens- und Lernumgebung, wie sie auch im hier vorgestellten Modell des persönlichen Wissensmanagements mitgedacht ist. Technisch läuft eine PLE auf (Web-)Applikationen hinaus, die für eine individuelle und dezentrale Zusammenstellung vieler verschiedener (Social-Software-)Werkzeuge [. . . offen ist und dem Lernenden im Idealfall lebenslang und unabhängig von bestimmten Bildungsinstitutionen zur Verfügung steht « (REINMANN 2008b, S. 55).

Persönliches Wissensmanagement hängt nach ReINMANN also eng mit selbst organisiertem Lernen zusammen und es erscheint nahe liegend, das Konzept PLE auch als Plattform für persönliches Wissensmanagement einzusetzen. Wenn man den Begriff des persönlichen Wissensmanagements jedoch (auch) für die Verwaltung von Zugangsdaten, von Links zu den beliebten Online-Shops oder für die Ablage des Schriftverkehrs benutzt, ist der Zusammenhang nicht mehr sehr eindeutig. Umgekehrt zeigen diese Beispiele auch, dass PLE, wenn sie tatsächlich in den normalen Arbeits- und Lebensalltag integriert werden sollen, auch solche »normalen «, nicht primär lernbezogenen Erfordernisse des persönlichen Wissensmanagements berücksichtigen sollten. Anwendungen, die dezidiert das Lernen unterstützen, sollten beispielsweise in der Lage sein, Tipps für die nächsten Lernschritte (-ressourcen) zu geben, die zum jeweiligen Vorwissen und zu den Interessen der Lernenden passen.

\subsection{PLE und E-Portfolio}

Die Arbeit mit elektronischen Portfolios kann auf eine lange Tradition papierbasierter Portfolio-Arbeit zurückgreifen (siehe dazu HORNUNG-PRÄHAUSER et al. 2007): In mehreren reformpädagogischen Ansätzen werden Lernzuwächse und Wissen von Schüler/-innen nicht (allein) durch schriftliche oder mündliche Wissensabfragen festgestellt, sondern mit Hilfe von ausgewählten Arbeiten der Schüler/-innen dokumentiert. Portfolio-Arbeit umfasst jedoch nicht allein die Dokumentation von (ausgewählten) Kompetenzen, sondern beinhaltet auch die mit anderen oder einem Tutor festgelegten Lernziele, Lernschritte oder -strategien und Dokumente, die sich auf das Lernen selbst beziehen, beispielsweise Einträge in einem Lerntagebuch. Als wesentliche Prozesse der Portfolioarbeit können demnach die Klärung der Zielsetzung und des Kontextes der Portfolio-Arbeit, das Sammeln, Auswählen und Verknüpfen von Dokumenten (Lernbelegen) mit dem Lernziel, das Reflektieren und Steuern des Lernprozesses, das Präsentieren und Weitergeben ausgewählter Dokumente sowie das Bewerten und Evaluieren von Lernprozessen/des Kompetenzaufbaus betrachtet werden.

Bei der E-Portfolio-Arbeit werden auch elektronische Medien genutzt: Sie werden bei der Erstellung, Dokumentation, Verwaltung und Präsentation von Portfolios genutzt. Diese neue Methode wird unter anderem »E-Portfolio«, »electronic portfolio« oder »digitales Portfolio« genannt. Vorteile und Mehrwert der E-Portfolio-Arbeit ergeben sich unter anderem im Hinblick auf die Dokumentation und Verwaltung der Dokumente, d. h. auch ihrer unterschiedlichen Versionen, sowie durch den erleichterten Zugriff und die Möglichkeit, auch Audio- oder Videodateien einzubinden. Die erleichterte Zugänglichkeit des E-Portfolios bietet Potenzial für eine intensivere Rückmel-

Portfolio-Arbeit

E-Portfolio-Arbeit 
dung und Lernprozessentwicklung und für einen Austausch zwischen Lernenden, auch über Instituts- und Klassengrenzen hinaus. Die Verwendung von Software beeinflusst sowohl das Portfolioprodukt als auch die Prozesse: Der Einsatz von Web-2.0-Technologien, insbesondere Social-Software-Technologien erweitert den Erstellungs-, Feedback- und Präsentationsspielraum erheblich.

Es zeigt sich, dass die E-Portfolio-Arbeit maßgeblich das aktive, selbst bestimmte und selbst gesteuerte Lernen von Lernenden unterstützt. Die Einführung der E-Portfolio-Arbeit stellt so einen wichtigen Schritt in Richtung Realisierung des selbst organisierten Lernens und einen Schritt zur weiteren Stärkung der Autonomie der Lernenden dar. Damit verbunden ist auch ein Rollenwechsel der »Lehrenden «: Die (E-)Portfolio-Arbeit benötigt Coaches, Tutor/-innen und Berater/-innen, impliziert und benötigt also auch einen Wandel der Lehr- und Lernkultur. Es überrascht daher nicht sehr, wenn zwischen dem E-Portfolio-Ansatz und PLE eine enge Verbindung hergestellt wird. So schreibt Graham ATtwell in seinem Weblog: »Ein E-Portfolio ist der Ort der Reflexion, des Erkennens von Lernen und seiner Präsentation. Eine PLE kann als Werkzeug (oder als ein Set von Werkzeugen) betrachtet werden, um nicht nur das Lernen zu präsentieren, sondern auch (individuell oder kollektiv) eine Darstellung umfangreichen Wissens" (ATTwell 2008, eigene Übersetzung).

Steven Downes nimmt wiederum Bezug auf einen (anderen) Beitrag von ATTwELL und stellt ihn unter die Überschrift: »E-Portfolios - the DNA of Personal Learning Environment? « (Downes 2007). Auch wenn es offensichtlich erscheint, dass PLE eine geeignete technologische Realisierung für die Arbeit mit E-Portfolios darstellen kann, erscheint dieses Bild von E-Portfolios als genetische Grundstruktur von PLE schief. Zudem zeigt sich deutlich, wenn man vorhandene E-Portfolio-Software und -anwendungen betrachtet, welches Merkmal in den bisherigen Beschreibungen des Konzepts PLE fehlt: Für die Praxis der E-Portfolio-Arbeit spielen die unterschiedlichen Sichten auf das E-Portfolio - was sieht der Lerner, der Tutor, der externe Begutachter? - eine große Rolle, die bei PLE-Konzeptionen bisher keine besondere Rolle spielt. Zudem wird E-Portfolio-Arbeit und -Software in der Regel zentral von Bildungseinrichtungen initiiert und gesteuert, das PLE-Konzept sieht jedoch auch dies vorrangig in den Händen der Lernenden liegend.

\subsection{PLE und „Communities of Practice»}

Ein weiteres Konzept, das im Zusammenhang mit PLE gebracht werden kann, ist das der »Community of practice « (CoP). Etienne Wenger und Jean LAVE identifizierten unter diesem Begriff ganz spezifische Arten von Zusammenschlüssen von Personen, die an einem gemeinsamen Thema interessiert sind. Die folgenden drei Elemente konstituieren eine CoP: (a) ein von allen geteiltes Thema oder Interesse, bei dem die Beteiligten nicht notwendigerweise die anderen kennen müssen, (b) das Engagement in gemeinsamen Aktivitäten und Diskussionen, sich gegenseitig zu helfen und Informationen zu teilen, und (c) die Gegenwart von Praktikern und die Entwicklung eines geteilten Repertoires von Quellen, wie Erfahrungen, Geschichten, Werkzeugen und Wegen, wie man mit wiederkehrenden Problemen umgeht (WENGER 2004). Die parallele Entwicklung dieser Elemente führt zu einer CoP. Da darin ein höchst fruchtvoller Austausch von Expertenwissen stattfindet, fand dieses 
Konzept auch großen Anklang in Unternehmen, die versuchten, solche CoP aktiv zu initiieren und zu pflegen, um dadurch das organisationale und individuelle Lernen zu unterstützen.

Mit dem Aufkommen der Anwendungen der Social Software wurde versucht, das Konzept wiederum auf das Web zu übertragen. Die Arbeit von LAvE und WeNGER ermöglicht nicht nur Hinweise auf typische Stadien von CoP, sondern gibt auch dezidiert Hinweise auf das dahinter stehende Lernkonzept: Lernen wird nicht als etwas beschrieben, das »im Kopf «stattfindet, losgelöst von anderen, sondern wird als maßgeblich beeinflusst und angeregt durch die Kommunikation betrachtet. Lernen ist nach dieser Sichtweise die Beziehung zwischen den Leuten: Lernen gehört gewissermaßen nicht zu den individuellen Personen, sondern zu den vielfältigen Konversationen, an denen sie teilnehmen (vgl. SMITH 2003).

In diesem Kontext erscheinen PLE, wenn man sie als persönliche Zentrale für aktive Beteiligte an CoP nutzt, als Ausgangspunkt für die Teilnahme am sozialen Austausch, z. B. durch das Kommentieren von Weblog-Beiträgen. PLE können also auch das technologische Konzept für diejenigen sein, die diese Form des Lernens in Web aktiv unterstützen wollen. Als Herausforderungen ergeben sich für das PLE-Konzept, dass jede/r Beteiligte normalerweise nicht nur Mitglied einer CoP ist, sondern sich häufig mehreren Arbeitgemeinschaften und Netzwerken zugehörig fühlt, auch solchen, die dem Typus CoP nicht exakt entsprechen.

\section{$7 \quad$ Ausblick}

Dieser Beitrag beschreibt das technologische Konzept Persönlicher Lernumgebungen, vorhandene Realisierungen und auch Abgrenzungen und Unterschiede $\mathrm{zu}$ (didaktischen) Methoden und andere technologische Konzepte des Lehrens und Lernens.

Dabei zeigt sich, dass das PLE-Konzept zu neuen Möglichkeiten, aber auch Herausforderungen führt. Besonders die graduelle Integration dieses Ansatzes in klassische E-Learning-Angebote birgt didaktisches Innovationspotenzial. So wird es besonders interessant sein zu sehen, inwieweit das selbst organisierte Lernen und die Möglichkeiten der damit verbundenen Kompetenzentwicklung auch wirklich durch didaktisch offenere Ansätze ergänzt werden. Mit der Einführung eines PLE-Konzepts ist ohne Frage der Wechsel von eher statischem und lehrerzentriertem Unterricht zu eher lernerorientierten Angeboten in sozialer Eingebundenheit notwendig. Dabei wurde auch angesprochen, dass PLE nicht für jede Zielgruppe und jedes Lern-Setting geeignet sind, da sie hohe Kompetenzen im Bezug auf Selbstorganisation und Medienkompetenz erfordern.

Aus unserer Sicht gehen das Konzept PLE und entsprechende Realisierungen, trotz dieser Herausforderungen, einen wichtigen Schritt zu mehr lernerzentrierten Angeboten, eben weil sie den Lernenden, seine Bedürfnisse und seine Selbstorganisation in den Mittelpunkt stellen. So wie mit LMS herkömmliche, traditionelle Modelle des Unterrichtens auf das Internet übertragen wurden, erwarten wir von den Realisierungen von PLE, dass damit geeignete Werkzeuge geschaffen werden, die offene und lernerzentrierte Unterrichtspraktiken, wie z. B. Webquests, die E-Portfolio-Methode oder Projektarbeit unterstützen und forcieren, eben weil damit herkömmliche Prinzipien des
Sozialer Austausch

Innovationspotenzial

Neue Lernkulturen 
Lehrens, wie die zentrale Stellung der Lehrenden als Wissensvermittler, nicht unterstützt werden und damit andere Lernkulturen, wie der Rollenwechsel von Lehrenden zu Lerntutoren, auch beim technologieunterstützten Lernen eine weite Verbreitung finden.

Ganz nebenbei ermöglichen uns gelungene Realisierungen von PLE auch die technologische Unterstützung, Organisation und Dokumentation von Wissens- und Kompetenzerweiterungen im Internet, die auch beiläufig oder im Arbeitsprozess stattfinden. Das bedeutet auch, dass PLE die Fokussierung und Konzentration auf zentrale Interessen, Arbeitsgebiete und Ressourcen ermöglichen, deren Vielzahl im Internet sonst zu Symptomen wie Informationsüberflutung führt und uns leicht den Überblick bzw. den Blick auf das Wesentliche verlieren lässt.

Förderhinweis

Die Arbeit an diesem Beitrag wurde zum Teil aus Mitteln des 6.

Forschungsrahmenprogrammes der Europäischen Kommission

(Schwerpunkt IST/Technology-Enhanced Learning) im Rahmen des

Integrierten Projektes TENCompetence (Vertragsnummer 027087)

ermöglicht (http//www.tencompetence.org). 


\section{Literaturhinweise}

Attwell, G.: The Personal Learning Environments - the future of eLearning?, in: eLearning Papers, 2007, 2 (1). Online zugänglich unter: http:// www.elearningeuropa.info/files/media/media11561.pdf (Abruf am 03.10.200).

Attwell, G.: What is the difference between an e-Portfolio and a Personal Learning Environment?, Weblogeintrag vom April 2008. Online zugänglich unter: http://www.pontydysgu.org/2008/04/what-is-the-difference-between-an-e-portfolio-and-a-personal-learning-environment/ (Abruf am 30. 10. 2008).

Bremer, H./Bittlingmayer, U. H.: Die Ideologie des selbstgesteuerten Lernens und die »sozialen Spiele« in Bildungseinrichtungen, in: Schulheft, 2, 2008.

DAALSGARD, C.: Social software: E-learning beyond learning management systems, in: European Journal of Open, distance and e-learning, 2006. Online zugänglich unter: http://www.eurodl.org/materials/contrib/ 2006/Christian_Dalsgaard.htm (Abruf am 04. 10. 2008).

Downes, S.: E-Portfolios - the DNA of the Personal Learning Environment?,

Weblogeintrag vom 25. 10. 2007. Online zugänglich unter: http://www. downes.ca/cgi-bin/page.cgi?post=42170 (Abruf am 30. 10. 2008).

Geser, G.: Open Educational Practices and Resources - OLCOS Roadmap 2012, Salzburg 2007. Online zugänglich unter: http://edumedia.salzburgresearch.at/images/stories/EduMedia/Inhalte/Publications/olcos_roadmap.pdf (Abruf am 04. 10. 2008).

Hilzensauer, W./SChaFferT, S.: Wikis und Weblogs bei SUN Microsystems: eine Erfolgsgeschichte eines Enterprise 2.0., in: Back, A./Gronau, N./TochterMANN, K. (Hrsg.): Web 2.0 in der Unternehmenspraxis. Grundlagen, Fallstudien und Trends zum Einsatz von Social Software, München 2008, S. 210-219.

Hornung-Prähauser, V./Geser, G./Hizzensauer, W./Schaffert, S.: Didaktische, organisatorische und technologische Grundlagen von E-Portfolios und Analyse internationaler Beispiele und Erfahrungen mit E-Portfolio-Implementierungen an Hochschulen, Salzburg 2007. Online zugänglich unter: http://edumedia.salzburgresearch.at/images/stories/e-portfolio_studie_srfg_fnma.pdf (Abruf am 04. 10. 2008).

KNowles, M.S.: Self-Directed Learning. A guide for learners and teachers. Prentice Hall/Cambridge 1975.

Liber, O./Johnson, M.: Special Issue on Personal Learning Environments. Interactive Learning Environments, 16, 1, 2008.

Olivier, B./Liber, O.: Lifelong learning: The need for portable Personal Learning Environments and supporting interoperability standards. Online zugänglich unter: http://wiki.cetis.ac.uk/uploads/6/67/Olivierandliber2001.doc (Abruf am 20. 10. 2008).

Reinmann, G.: Persönliches Wissensmanagement. Vortrag, Karlsruhe, Oktober 2008. Online zugänglich unter: http://medienpaedagogik.phil. uni-augsburg.de/denkarium/wp-content/uploads/2008/10/vortragpwm _karlsruheokt08.pdf (Abruf am 30. 10. 2008). 
Reinmann, G.: Lehren als Wissensarbeit? Persönliches Wissensmanagement mit Weblogs, in: Information - Wissenschaft \& Praxis, 2008, Heft 1, S. $49-57$.

SCHAFFERT, S.: Semantic Social Software: Semantically Enabled Social Software or Socially Enabled Semantic Web?, in: ReCh, J./DeCKer, B./Ras, E. (Eds.): Emerging Technologies for Semantic Work Environments: Techniques, Methods, and Applications. Hershey 2008.

SCHAFfert, S/Hilzensauer, W.: On the way towards Personal Learning Environments: Seven crucial aspects, in: eLearning Papers, 2008, 9. Online zugänglich unter: http://www.elearningpapers.eu/index.php?page=doc\& doc_id=11938\&doclng $=6 \& v o l=9$ (Abruf am 30. 10. 2008).

Schulmeister, R.: Gibt es eine Net Generation?, Work in Progress, Hamburg 2008. Online zugänglich unter: http://www.zhw.uni-hamburg.de/pdfs/ Schulmeister_Netzgeneration.pdf (Abruf am 05. 06. 2008).

SмITH, M. K.: Communities of practice, in: the encyclopedia of informal education, 2008. Online zugänglich unter: www.infed.org/biblio/communities_of_practice.htm (Abruf am 28. 10. 2008).

Turner, R.: A Conceptual Map of the Social Web, Weblogeintrag vom 4. 6. 2008. Online zugänglich unter: http://www.websocialarchitecture.com/community/enterprise/ (Abruf am 30. 10. 2008).

WENGER, E.: Communities of practice - a brief introduction, 2004. Online zugänglich unter: http://www.ewenger.com/theory/ (Abruf am 17. 10. 2008).

WIKIPEDIA: History of personal learning environments. Online zugänglich unter http://en.wikipedia.org/wiki/History_of_personal_learning_environments (Abruf am 14. 10. 2008).

Wild, F./Kalz, M./Palmer, M. (Eds.): Proceedings of the First International Workshop on Mashup Personal Learning Environments (MUPPLE08) September, 17, 2008, Maastricht, NL, in: CEUR Workshop Proceedings. Online zugänglich unter: http://ceur-ws.org/Vol-388 (Abruf am 13. 10. 2008).

WiLD, F./MöDritscher, F./Sigurdason, S.: Designing for Change: Mash-Up Personal Learning Environments, in: eLearning Papers, 9, Juli 2008. Online zugänglich unter: http://www.elearningpapers.eu/index.php?page= doc\&doc_id=11939\&doclng=3 (Abruf am 04. 10. 2008) 